\title{
GeoAI for Topographic Mapping and the Intelligent National Map
}

\author{
E. Lynn Usery \\ U.S. Geological Survey
}

usery@usgs.gov

Keywords: GeoAI, feature extraction, intelligent National Map

\begin{abstract}
:
Geospatial Artificial Intelligence (GeoAI) is the application of artificial intelligence (AI) methods and techniques to geospatial data, processes, models, and applications. GeoAI methods are being used by the U.S. Geological Survey (USGS) to extract and model topographic features and relationships. Specifically, the USGS has investigated and developed applications in terrain feature extraction, hydrographic network extraction, and semantic modelling. Deep learning (DL) methods are being used with high spatial resolution lidar and digital elevation data to extract hydrography and terrain elements. Other data sources include nationwide hydrography, geographic names, historical topographic maps, and other datasets from the National Map.
\end{abstract}

The GeoAI methods are a part of an approach to develop an intelligent National Map. An intelligent National Map requires the use of GeoAI research and implementation to be capable of feature extraction, representation, knowledge construction and automated logic and inference from the geospatial data. The framework datasets include the 3D Elevation Program (3DEP), the National Hydrography Dataset (NHD) and the other nationwide datasets that are parts of the National Map. These data provide the base data and training samples for the deep learning process.

Additional research is to represent and structure the data and extracted features using a semantic representation based on the Resource Description Framework data model. This representation supports logic and inference through the Web Ontology Language and a concept and implementation of the Map as a Knowledge Base has been developed for the National Map. The goal is the development of an intelligent National Map using GeoAI and the semantic representation.

The implementation of GeoAI relies on a high-performance computing (HPC) infrastructure with the DL and other methods implemented on supercomputers. These computers use clusters of processors and Graphics Processing Units (GPUs) with thousands of cores in a distributed configuration. The HPC environment is required to handle the large data volumes and computational requirements of GeoAI.

This presentation is a documentation of the recent work and current state of this research and an attempt to help define the directions of research and applications in the field of GeoAI, specifically for topographic mapping within the USGS. 